\title{
Incidencia de los aspectos socioculturales en al autorregulación del aprendizaje y el rendimiento académico en estudiantes de la básica secundaria Incidence of sociocultural aspects on self-regulation of learning and academic performance in junior high school students
}

\author{
Pola Yasmid Oviedo Suárez ${ }^{1}$ \\ Félix Morales Sánchez ${ }^{2}$ \\ Francisco Conejo Carrasco ${ }^{3}$ \\ Jenny Consuelo Mahecha Escobar ${ }^{4}$ \\ Corporación Universitaria Minuto de Dios \\ Sede principal Bogotá, Colombia
}

\begin{abstract}
1 https://orcid.org/0000-0002-1371-6564 Docente de aula en la Institución Educativa Cirales, El Carmen Santander, Colombia fandy06@hotmail.com 3133876759

2 https://orcid.org/0000-0003-4002-8043 Docente de aula en la Institución Educativa Cirales, El Carmen Santander, Colombia moralesan145@hotmail.com3105858076
\end{abstract}

3 https://orcid.org/0000-0002-3490-1937 Docente investigador Maestría de Educación UNIMINUTO, Colombia fconejocarr@uniminuto.edu.co 3153482752

4 https://orcid.org/0000-0002-9799-7200 Docente tutora de proyectos de investigación Maestría de Educación, UNIMINUTO, Colombia. ymahech1@uniminuto.edu.co 3102156451315

\section{RESUMEN}

El fenómeno del rendimiento académico ha sido objeto de innumerables investigaciones en el ámbito educativo, dada su complejidad y maneras de interpretar su dinámica en el estudiantado. Por ende, cada institución educativa tiene la responsabilidad de reconocer las necesidades y expectativas de la comunidad educativa y sus entornos, de tal forma que se ofrezca una educación contextualizada. Es por ello que el objetivo general de la presente investigación se orienta hacia el análisis de la incidencia de los factores socioculturales de los estudiantes de básica secundaria, sede A y 
sede $\mathrm{K}$ de la Institución Educativa Cirales en la autorregulación del aprendizaje y el rendimiento académico.

En el diseño metodológico se adoptó el enfoque cualitativo de tipo descriptivo apoyado en instrumentos de recolección denominados grupo focal, entrevista semiestructurada y análisis documental, para ser aplicados en una muestra de 16 estudiantes, tres docentes y rectora, pertenecientes a la sede $\mathrm{A}$ y sede $\mathrm{K}$. El análisis y discusión de resultados se llevó a cabo a partir de la descripción y comparación del marco referencial con los hallazgos en las sedes muestreadas. Por último, se definieron las conclusiones correspondientes al cumplimiento del objetivo principal y específicos. En ese orden de ideas, se determinó que los aspectos que influyen en la autorregulación del aprendizaje y el rendimiento académico emanan de la presencia de hogares disfuncionales, falta de transporte escolar, existencia de noviazgos en la pre adolescencia, uso inadecuado del celular, influencia de terceros hacia el consumo de sustancias psicoactivas y falencias en la mediación docente y estudiante.

PALABRAS CLAVE: Aspectos socioculturales, aspectos escolares, autorregulación del aprendizaje, rendimiento académico.

\section{ABSTRACT}

The academic achievement has been the subject of countless investigations in the educational field, given its complexity and ways of interpreting its dynamics in the student body. Therefore, each educational institution has the responsibility to recognize the needs and expectations of the educational community and its environments, in such a way that a contextualized education is offered. And because of this the general objective of this research is oriented towards the analysis of the incidence of sociocultural factors of high school students, campus $A$ and campus $\mathrm{K}$ of the Cirales Educational Institution in the self-regulation of learning and academic performance.

In the methodological design, the qualitativedescriptive approach was made and supported by collection instruments called focal group, semistructured interview and documentary analysis, to be applied in a sample of 16 students, three teachers and principal, belonging to campus $A$ and campus $\mathrm{K}$. The analysis and discussion of the results was carried out from the description and comparison of the reference framework with the findings in the sampled sites. Finally, the conclusions corresponding to the achievement of the main and specific objectives were defined. In that order of ideas, it was determined that the aspects that influence the self-regulation of learning and academic performance comes from the presence of dysfunctional homes, lack of school transportation, existence of dating in preadolescence, inappropriate use of cell phones, influence towards the usenes of psychoactive substances and deficiencies in teacher and student mediation.

\section{KEY WORDS}

Sociocultural aspects, school aspects, selfregulation of learning, academic performance.

\section{INTRODUCCIÓN}

El concepto de rendimiento académico con el transcurrir del tiempo ha ido evolucionando, en parte por la comprensión que se ha adquirido en el campo educativo al darle al estudiante el rol de eje central del sistema, que cada día tiene el reto de pasar por completo de la pedagogía tradicional y avanzar a aquella que le permite al estudiante construirse así mismo de acuerdo a su contexto.

En tal sentido, Meneses et al. (2013) atribuyen a las políticas educativas establecidas por determinados países, el fracaso de muchas estrategias para alcanzar la calidad educativa, dado que no tienen en cuenta la realidad de 
las comunidades educativas, al respecto de sus necesidades y expectativas, en especial los contextos rurales. En efecto, los aspectos vinculados al rendimiento académico otorgan sentido a la praxis pedagógica y orientan aspectos curriculares.

Los establecimientos educativos requieren dar mayor importancia a la comprensión de los aspectos que pongan en riesgo el rendimiento académico, porque también se asocia con la problemática del ausentismo escolar. El conocimiento del contexto real del estudiantado debe convertirse en la base para el direccionamiento de políticas educativas fundamentadas en las particulares de las comunidades.

En ese orden de ideas, es conveniente indagar sobre situaciones cotidianas vivenciadas por distintos miembros de la comunidad educativa, desde aspectos externos e internos que, en dado momento, inciden en el rendimiento académico y el aprendizaje autorregulado. En el ámbito educativo, dichos aspectos definen los ambientes de aprendizaje que toda institución requiere conocer y valorar para comprender la realidad de sus entornos en la búsqueda de una educación contextualizada.

De tal manera que los aspectos socioculturales son catalogados como factores extrínsecos que intervienen en la motivación, hacia el acto de aprender, del mismo modo que en el rendimiento académico. Jurado (2012) resalta la significación de las pautas de crianza propias de cada región como determinantes en la caracterización familiar. De hecho, los estudiantes van formando su identidad de acuerdo al contexto, donde adquieren desde niños, hábitos que moldean sus creencias frente al aprendizaje dentro y fuera del entorno escolar. Asimismo, estos son considerados de carácter familiar, social y socioeconómico; mientras que los aspectos escolares, se pueden abordar desde las características que emergen de la mediación docente y estudiante, así como el clima y fracaso escolar.

Cabe considerar que, los factores familiares y su correspondencia con el rendimiento académico han sido investigados por Tilano, Henao, y Restrepo (2009) con aportes significativos, ya que evaluaron algunas prácticas educativas familiares a través de variables que conllevan a determinar que los estudiantes formados en familias autoritarias y rígidas obtienen rendimientos académicos favorables, de manera opuesta aquellos que conviven con familias permisivas de poca exigencia se distinguen por un desempeño académico bajo.

Mientras que, los aspectos sociales son factores externos donde los estudiantes adoptan modelos, con el propósito de recibir aceptación en algunos grupos de amigos, en otras, por mostrar independencia o libertad y que traen como consecuencia desmotivación por el estudio y ausentismo escolar. En las zonas rurales el aspecto social influye en los estudiantes desde la cultura campesina, como consecuencia de que las familias presentan limitaciones como la desigualdad, pobreza y el bajo nivel educacional de los padres, que conlleva al poco grado de acompañamiento y asesoría de tareas y responsabilidades académicas de los hijos.

Además, los grupos de amigos con los cuales interactúan los estudiantes se perfilan como un aspecto social causante de rendimiento académico deficiente, ocasionado por el uso inadecuado de las redes sociales y la distracción que causan los celulares dentro y fuera del aula, en ello los adolescentes invierten gran parte del tiempo. Lo anterior conlleva a conductas inapropiadas, desinterés, indisciplina y pérdida académica y deserción escolar (Meneses et al., 2013).

Narváez y Oyola (2015) por su parte, consideran que los adolescentes tienden a imitar algunas conductas presentes en las comunidades, 
sin embargo, si en esos ambientes existen problemáticas sociales como el alcoholismo, consumo de alucinógenos, pandillismo, entre otros, estos pueden entorpecer el rendimiento académico de los educandos.

Por otra parte, los aspectos escolares están vinculados a la vida estudiantil, por ello se relacionan con el clima o ambiente escolar, pérdida escolar y las mediaciones de diferente índole que convergen en las instituciones educativas. De ahí, que pueden influir en el rendimiento académico del estudiantado actuando como limitantes de la instrucción docente y familiar. Por consiguiente, es relevante tener presente que, de acuerdo con la actitud que asume el docente frente a la praxis educativa, ésta se reflejará en el rendimiento académico del estudiantado. Parra (2014) concluye que un aspecto a tener en cuenta, al planear la instrucción, es la creación de un ambiente de aula que permita la reflexión con respecto al aprendizaje adquirido, acompañando todo proceso de enseñanza de una motivación extrínseca. De igual forma, el producto de la mediación desde lo social y cultural debe ser reflejado en un buen desempeño académico en el estudiantado.

Ahora bien, el reconocimiento de los factores intrínsecos también facilita la interpretación de los aspectos internos vinculados al rendimiento académico, ya que son propios de cada estudiante. Por esa razón, en los entornos educativos es cada vez más frecuente el desarrollo de proyectos que tienen como propósito la caracterización del aprendizaje autorregulado, con miras al establecimiento de sus aportes a la calidad educativa. (Schunk, 2012) lo define como aquel conjunto de conductas de autorregulación que regulan un comportamiento autónomo hacia el logro de metas, involucrando, además, variables cognoscitivas y afectivas.

Por último, es conveniente acotar, que la metacognición de la cual se deriva la autoobservación, se presenta como estrategia para el aprendizaje autorregulado, así lo sostiene Torrano, Fuentes, y Soria (2017) al considerar que en ésta se incluyen actividades donde el estudiante toma conciencia del estado de su cognición, motivación, afecto, uso del tiempo, esfuerzo y las condiciones de la tarea y el contexto. Por consiguiente, es la herramienta que utiliza el educando para modificar aspectos extrínsecos e intrínsecos para favorecer su aprendizaje.

El presente trabajo investigativo, pone de manifiesto el interés por resolver la pregunta de investigación, ¿Cuál es la incidencia de los aspectos socio-culturales de los estudiantes de la básica secundaria sede $A$ y sede $K$ de la institución educativa Cirales en la autorregulación del aprendizaje y el rendimiento académico?, para llegar a la comprensión y reflexión sobre el estudio de dicho fenómeno, así como ampliar la experiencia en el campo profesional mediante la puesta en práctica de un enfoque cualitativo, reflejado en la recolección, condensación e interpretación de los resultados encontrados en cada categoría de investigación.

\section{MATERIALES Y MÉTODOS}

La naturaleza de la presente investigación corresponde a un enfoque cualitativo de tipo descriptivo, partiendo de la inspección de aspectos particulares hasta alcanzar aspectos generales, por esa razón se evidencia la indagación de detalles y acciones presentadas en la cotidianidad de las personas que integran la muestra. Quecedo y Castaño (2003), sostienen que "el diseño cualitativo facilita una recogida de datos empíricos, que ofrecen descripciones complejas, de acontecimientos, interacciones, comportamientos, pensamientos, que conducen al desarrollo o aplicaciones de categorías y relaciones que permiten la interpretación de los datos. 
La población del estudio la conforman 110 estudiantes de la básica secundaria de la Institución Educativa Cirales, los cuales están distribuidos así: 75 estudiantes en la sede $A$ y 35 estudiantes en la sede $\mathrm{K}$, con edades correspondientes a los 11 y 15 años.

\section{MUESTRA (PARTICIPANTES)}

El criterio para la selección de la muestra obedeció a la selección de un muestreo de avalancha o bola de nieve, dado que permitió la interacción de los educandos. Crespo y Salamanca (2007) afirman que esta clase de muestreo es más práctico y eficiente que el realizado por conveniencia, gracias a la presentación que hace el sujeto ya incluido en el proyecto, con lo cual resulta más fácil establecer una relación de confianza con los participantes.

La muestra está conformada por la rectora de la Institución, 8 estudiantes, 2 de cada grado de básica, dos docentes quienes orientan las asignaturas de humanidades y lengua castellana y educación física, pertenecientes a la sede $A$. En la sede K, compuesta por 8 estudiantes, 2 de cada grado de básica y 1 docente que orienta la asignatura de ciencias sociales.

\section{INSTRUMENTOS}

Para el alcance de los objetivos propuestos en el estudio, fueron establecidas cuatro categorías de investigación correspondientes con los objetivos específicos, de igual manera, fueron seleccionados y aplicados los siguientes instrumentos de recolección de información.

\section{- Grupo focal a estudiantes "dialoguemos} sobre aprendizaje autorregulado" (Hernández, Fernández, y Baptista, 2010) afirman que los grupos focales consisten en reuniones de personas con pocos integrantes, quienes conversan en torno a uno o varios temas en un ambiente relajado e informal, bajo la conducción de un especialista en dinámicas grupales. Con este método se logró intercambiar opiniones, sentimientos, vivencias, creencias y prácticas propias del entorno sociocultural de los estudiantes de básica secundaria.

Para la aplicación en campo de dicho instrumento fueron organizados grupos de 8 estudiantes en cada una de las sedes, dos estudiantes por grado de la básica secundaria, recurriendo a un protocolo con 12 preguntas principales y tres o cuatro secundarias, además, durante la entrevista se formularon nuevas preguntas que dependieron de las respuestas que iban dando los estudiantes y otras que podrían ser significativa para la investigación.

Con el grupo focal, considerado también, como una entrevista grupal semiestructurada, pudieron establecerse hallazgos significativos correspondientes a la categoría 1: aspectos externos de tipo sociocultural de los estudiantes de básica secundaria de la sede $A$ y sede $K$, así como a la categoría 3: aspectos socioculturales que afectan el rendimiento académico de los estudiantes de básica.

\section{- $\quad$ ANÁLISIS DOCUMENTAL}

Dicho instrumento fue aplicado desde la revisión e interpretación de los datos correspondientes al cuadro de promoción del año 2019, de los estudiantes muestreados. De acuerdo con los desempeños académicos encontrados en el análisis documental se avanzó a la caracterización del rendimiento académico, teniendo en cuenta, además, detalles encontrados en el grupo focal y que aportaban información valiosa a la categoría 2: aspectos externos de tipo escolar relacionados con el rendimiento académico de los estudiantes de básica secundaria sede $A$ sede $K$.

\section{- $\quad$ ENTREVISTA: "A FORMULAR ESTRATEGIAS EN BÚSQUEDA DE UN APRENDIZAJE AUTORREGULADO"}

Otro instrumento de recolección utilizado fue la entrevista, dada su flexibilidad y dinámica; se 
favoreció con ella la comunicación con docentes y rectora a partir de preguntas que respondían a unos temas previamente establecidos de acuerdo a los aspectos sobre los que se quería investigar. En relación a lo anterior (Hernández et al., 2010) afirman que esa clase de entrevista se basa en una guía de asuntos o preguntas y el entrevistador tiene la libertad de introducir interrogantes adicionales para precisar conceptos $\mathrm{u}$ obtener mayor información sobre los temas deseados.

A partir de la aplicación del formato de entrevista a docentes y directivo, se obtuvieron datos pertenecientes a la categoría 4 denominada: aspectos internos relacionados con estrategias que aportan al mejoramiento del rendimiento académico y autorregulación del aprendizaje.

Por consiguiente, antes de llevar a cabo el trabajo de campo, fue necesaria la validación de los instrumentos por parte de un experto en educación, con el propósito de hacer ajustes respectivos en la estructura de las preguntas del grupo focal y entrevista como tal.

Debe señalarse, que previamente al procesamiento y análisis de datos, se utilizó una estrategia de condensación de información denominada matriz de categorización, donde se plasmaron los hallazgos de cada categoría, de acuerdo con el instrumento aplicado.

\section{RESULTADOS y DISCUSIÓN}

Los hallazgos obtenidos al aplicar el grupo focal facilitaron el alcance del primer objetivo específico, relacionado con la descripción de aspectos socioculturales de los estudiantes de básica. En la tabla $\mathrm{N}^{\circ} 01$ se muestran los resultados de la categoría 1: aspectos externos de tipo sociocultural de los estudiantes de básica secundaria de la sede A y sede $\mathrm{K}$, los cuales fueron abordados desde los aspectos familiares, sociales y socioeconómicos. 
Tabla $\mathrm{N}^{\circ} 01$

Aspectos externos desde el contexto sociocultural en la sede $A$ y la sede $K$

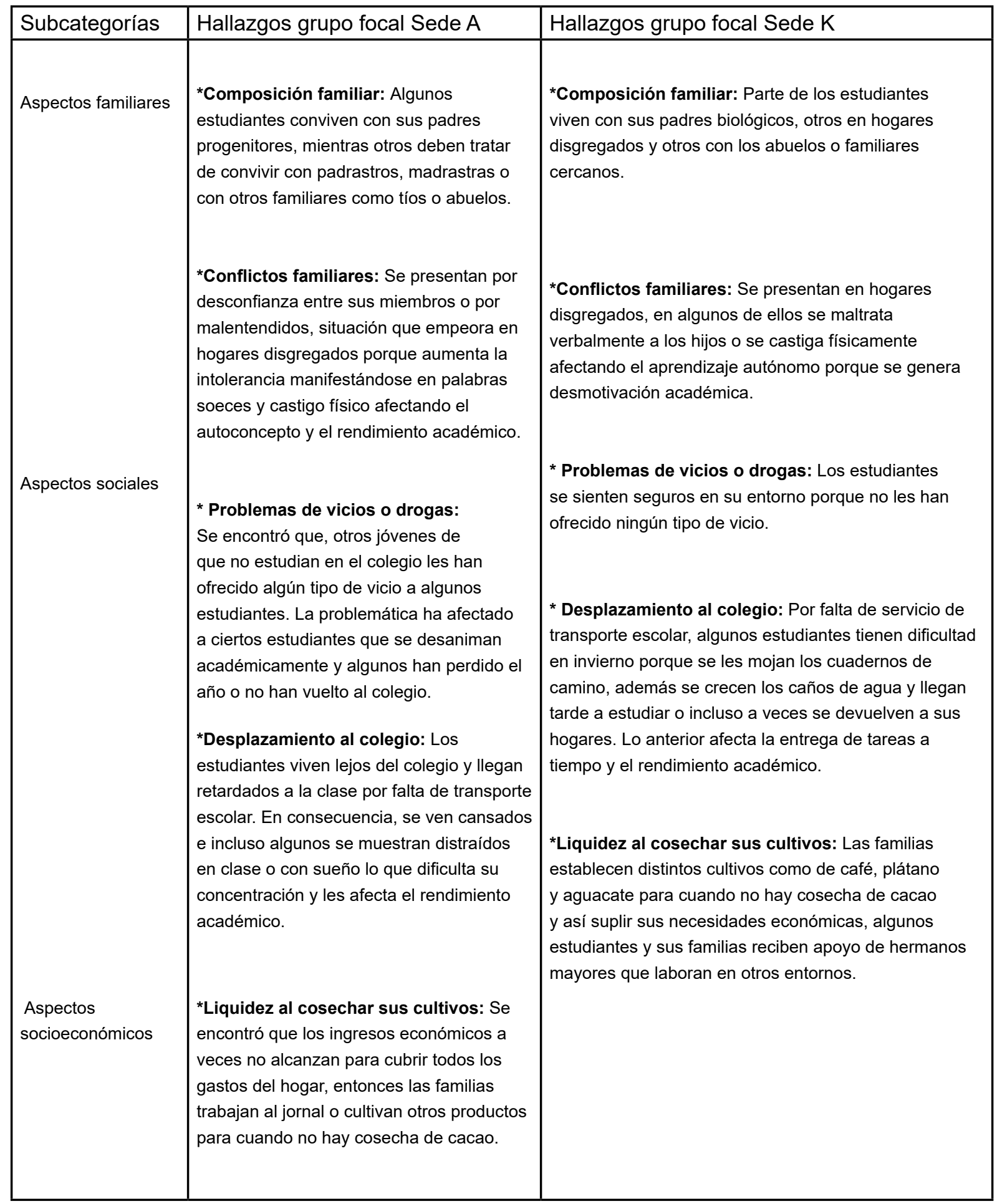

Fuente: Elaboración Propia 
En primer orden, el aspecto familiar, a partir del cual se pudo establecer que los estudiantes conviven en hogares funcionales y disfuncionales, en ambos se presentan conflictos, sin embargo, se agudizan cuando no hay tolerancia y comprensión entre alumnos y padrastros, situación que influye en el rendimiento académico, Gutiérrez (2014) sostiene que los estudiantes provenientes de hogares funcionales se muestran más seguros de sí mismos, tienen empatía y buena autoestima; mientras que los vienen de hogares disfuncionales, reflejan un rendimiento académico pobre, resultado de un bajo autoconcepto, inseguridad y violencia intrafamiliar.

En segundo orden, los aspectos sociales que inciden en el rendimiento académico y aprendizaje autorregulado de los alumnos de básica, son más notorios en la sede $A$, en relación a situaciones de convivencia en sus habitantes, en los cuales están presentes los estudiantes, además el consumo de alucinógenos que, siendo un tabú, no deja de ser un impedimento para el alcance de metas en los escolares. Según lo enunciado por Narváez Oyola (2015) el rendimiento académico se afecta negativamente porque los alumnos obtienen comportamientos inadecuados en la población donde interactúan. Al mismo tiempo, un aspecto social común que afecta en especial el rendimiento académico de las dos sedes es la falta de transporte escolar y el desplazamiento de los estudiantes al colegio en temporada de invierno.

En tercer orden, los aspectos socioeconómicos de los estudiantes de las sedes muestreadas poco inciden en el rendimiento académico y el aprendizaje autorregulado, dado que acuden a estrategias para establecer una proporción entre sus ingresos y egresos para el sustento del hogar. En este sentido, Jama y Cornejo (2016), afirma que cuando los ingresos económicos, resultado de la cosecha son suficientes se logra un equilibrio en sus servicios básicos, por esta razón, los padres se responsabilizan de la inversión que requiere el estudio de sus hijos.

En referencia al segundo objetivo específico, los resultados facilitaron la caracterización del rendimiento académico, por medio del análisis documental y el grupo focal; a partir de los cuales se encontraron detalles de aspectos externos de tipo escolar influyentes en el desempeño académico a saber: clima escolar, fracaso escolar, mediación docente y mediación del estudiante. En la tabla $\mathrm{N}^{\circ} 02$ se aprecian los resultados de la categoría 2: aspectos externos de tipo escolar relacionados con el rendimiento académico de los estudiantes de básica secundaria sede A sede $\mathrm{K}$. 
Tabla $\mathrm{N}^{\circ} 02$

Aspectos externos de tipo escolar de los estudiantes de básica sede $\mathrm{A}$ y sede $\mathrm{K}$

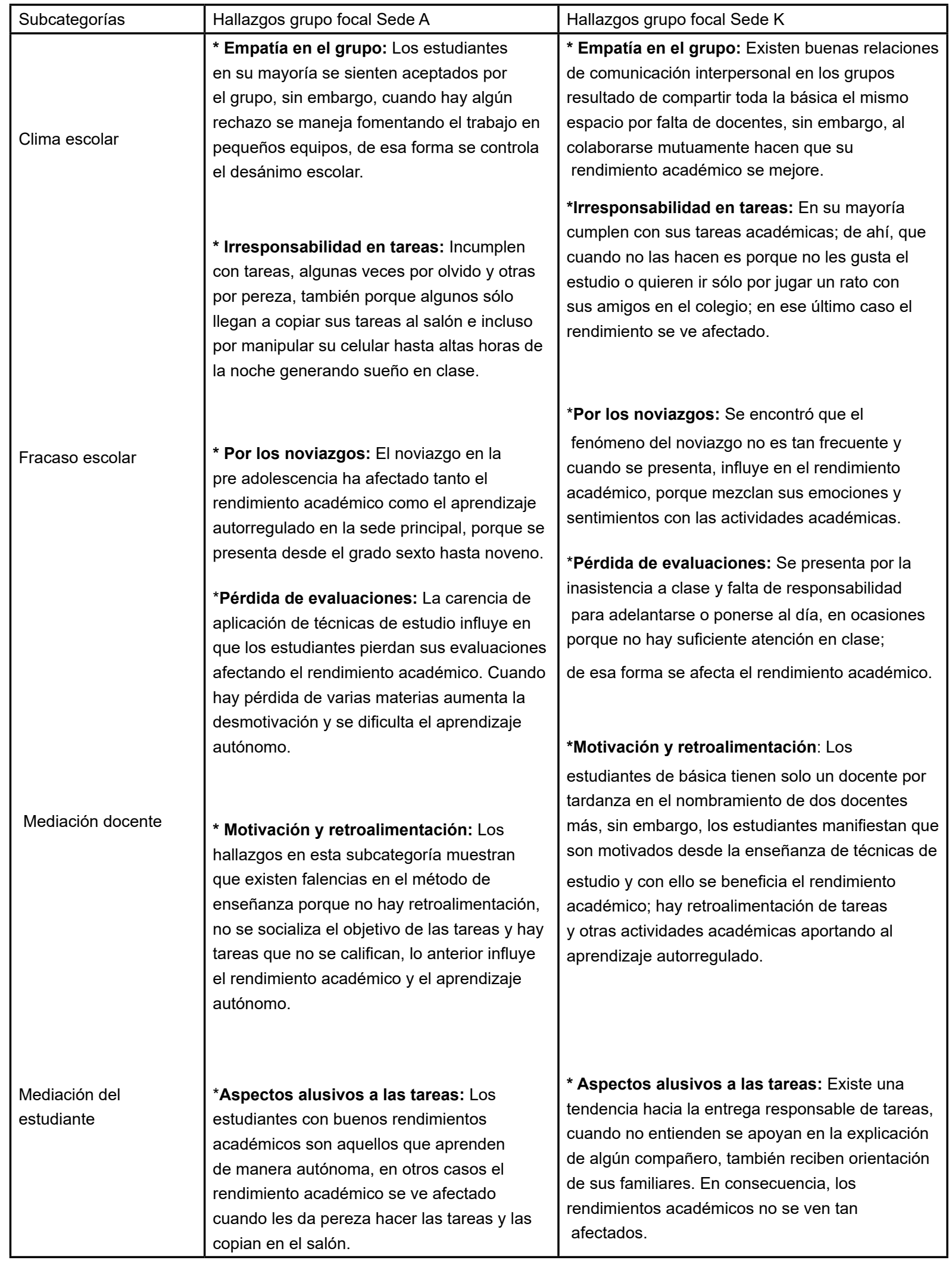


De acuerdo con lo anterior, los aspectos externos de tipo escolar inciden en el rendimiento académico y aprendizaje autorregulado son:

-Clima escolar: Los estudiantes de las sedes muestreadas señalaron ser aceptados por sus compañeros de grado, evidenciando empatía y lazos de confianza entre ellos, de ahí que favorece el rendimiento escolar. Se encontró congruencia con lo afirmado por Barreto y Álvarez (2017) al considerar que un buen clima escolar se evidencia desde la empatía que hay entre estudiantes y docentes.

-Fracaso escolar: Los hallazgos señalan que está asociado a la irresponsabilidad para entregar actividades, noviazgos en pre adolescencia y desempeños bajos por pérdida de evaluaciones y materias. Al respecto Luna (2018) hace referencia a que el fracaso escolar puede ser influenciado por factores externos de tipo social, familiar y escolar.

-La mediación docente: Los resultados obtenidos en la aplicación de los instrumentos en las sedes seleccionadas, indican que se presentan deficiencias en la sede $A$ en cuanto a la retroalimentación del aprendizaje, la socialización del objetivo de las tareas y la calificación de las mismas. Lo anterior provoca desmotivación académica en algunos estudiantes e incidencia en el rendimiento escolar y aprendizaje autónomo. Según Hernández (2017) la función del profesor es esencial en la orientación de los alumnos hacia el logro de sus metas.

-La mediación del estudiante: Debe señalarse, que, como producto de la entrevista grupal, en la sede principal, se encontraron dificultades para el aprendizaje autorregulado, como resultado de la frecuente copia de sus tareas, además de la falta de estrategias que conlleven al establecimiento de metas propias con las cuales le encuentren sentido a lo que aprenden. Mientras que en la sede $\mathrm{K}$, los estudiantes poseen un autocontrol de su rendimiento académico y logran adecuar los aspectos externos e internos para cumplir sus metas.

En este apartado, también fue condensada e interpretada la información documental, logrando establecer correspondencias entre los anteriores hallazgos y los desempeños académicos, conforme al cuadro de promoción escolar de 2019, donde se encontró que los estudiantes de la sede $\mathrm{K}$ obtuvieron mejores rendimientos académicos, mientras que en la sede $A$, se hallaron mayores porcentajes de estudiantes con desempeños básicos y bajos.

De acuerdo con la información anterior, se pudo establecer que, de los 8 estudiantes de la sede A muestreados en el grupo focal, obtuvieron en términos de porcentaje los siguientes desempeños académicos, 3,84\% desempeño bajo, 42,3\% desempeño básico, $48,07 \%$ desempeño alto y solo un $5,76 \%$ se obtuvo un desempeño superior. Asimismo, las ponderaciones académicas obtenidas por los estudiantes de la sede $\mathrm{K}$ fueron; desempeño bajo 0,97\%, con desempeño básico $25 \%$, estudiantes con un desempeño académico alto $44,23 \%$ y en desempeño superior $37,52 \%$; es de acotar que la escala valorativa de la institución se distribuye de la siguiente manera; desempeño Bajo 1 a 64, desempeño Básico 65 a 79, desempeño alto 80 a 94 y desempeño superior 95 a 100.

Por lo demás, el alcance del tercer objetivo específico contempló la comparación de la información proveniente del grupo focal, en cuanto a los aspectos socioculturales de la sede $A$ y sede $K$, y cómo estos influyen en el rendimiento académico. En efecto, se determinó que los aspectos socioculturales en la sede $A$ y sede $\mathrm{K}$, presentan semejanzas y diferencias incidentes en el rendimiento académico del estudiantado.

De acuerdo con lo afirmado por Muñoz et al. (2014) el desempeño académico es producto de 
la interrelación estudiante-cultura y escuela. Por consiguiente, el logro o pérdida académica de los estudiantes de la Institución, también va ligado a la identidad adquirida por los adolescentes, aspecto influenciado por particularidades del contexto sociocultural y socioeconómico.

Los resultados de la comparación en las sedes muestreadas arrojaron semejanzas, por ejemplo, desde los aspectos familiares por presencia de hogares disfuncionales y conflictos intrafamiliares, además de castigos físicos en algunos núcleos familiares. En el aspecto social hay congruencias en cuanto a la falta de transporte escolar, dificultades de desplazamiento en épocas de lluvias, influencias de terceros que los animan a no estudiar más. En el aspecto socioeconómico hay similitudes en las estrategias que usan las familias para mejorar sus ingresos económicos y apoyar a sus hijos.
En efecto, las diferencias desde los aspectos familiares, están asociados con la falta de mayor control o supervisión de padres a hijos en la sede A, de igual manera, en los aspectos sociales las mayores diferencias están en la presencia de problemáticas asociadas al consumo de sustancias psicoactivas, también en la sede $A$ y en lo socioeconómico, se estableció que hay mayor propiedad de las tierras en la sede $\mathrm{K}$.

Finalmente, una vez aplicados los instrumentos de entrevista a rectora y docentes pertenecientes a la muestra, al igual de los estudiantes que hicieron parte del grupo focal, se resaltaron hallazgos para el logro del cuarto objetivo específico, orientado a proponer estrategias que, de acuerdo con los aspectos socioculturales, aporten al mejoramiento del rendimiento académico y la autorregulación del aprendizaje. En la tabla $\mathrm{N}^{\circ} 03$ se muestran los resultados de la categoría 4: aspectos internos relacionados con estrategias que aportan al mejoramiento del rendimiento académico y autorregulación del aprendizaje. 
Tabla $\mathrm{N}^{\circ} 03$

Aspectos internos y estrategias para el rendimiento académico y la autorregulación del aprendizaje en la básica secundaria

\begin{tabular}{|c|c|c|}
\hline Subcategorías & Hallazgos grupo focal Sede A & Hallazgos grupo focal Sede K \\
\hline Metacognición & $\begin{array}{l}\text { *Entrevista: Tareas planeadas. Se } \\
\text { deben planear clases con objetivos claros } \\
\text { y dárselos a conocer a los estudiantes. } \\
\text { Se requiere estimular el aprendizaje } \\
\text { autónomo en los estudiantes. Grupo } \\
\text { focal: Se encontró que hay escasa } \\
\text { planeación de tareas y los alumnos no } \\
\text { cuentan con un cronograma para el } \\
\text { desarrollo de las mismas. } \\
\text { *Entrevista: Aprendizaje autónomo. } \\
\text { Hacer del ejercicio de las tareas un } \\
\text { proceso flexible y contextualizado. } \\
\text { La realización de un diagnóstico } \\
\text { socioeconómico y familiar para ver su } \\
\text { correlación con el acto de prender. } \\
\text { *Entrevista: Técnicas de inteligencia } \\
\text { emocional. Desde rectoría y personero } \\
\text { estudiantil se impulsa estrategia "levanta } \\
\text { la mano" que ha aportado a la reducción } \\
\text { de conflictos entre estudiantes. } \\
\text { *Grupo focal: Manejo de emociones. } \\
\text { Si los estudiantes aprenden de manera } \\
\text { autónoma, por lo general se sienten } \\
\text { satisfechos de alcanzar sus metas y rendir } \\
\text { académicamente. }\end{array}$ & $\begin{array}{l}\text { * Entrevista: Tareas planeadas. Es importante } \\
\text { enseñar buenos hábitos saludables, eso favorece } \\
\text { el aprendizaje autorregulado. Grupo focal: Los } \\
\text { educandos tienen un autocontrol en el desarrollo } \\
\text { de sus tareas favoreciendo su aprendizaje } \\
\text { autónomo. } \\
\text { *Entrevista: Aprendizaje autónomo. Formación } \\
\text { al estudiantado en su autodisciplina, esto les } \\
\text { ayuda a aprender de manera autónoma. } \\
\text { eEntrevista: Técnicas de inteligencia } \\
\text { emocional. Se encontró que existe conciencia al } \\
\text { respecto del apoyo psicosocial a los estudiantes } \\
\text { de parte de su docente. } \\
\text { * Grupo focal: Manejo de emociones. Tratan } \\
\text { de controlar sus impulsos y tranquilizarse } \\
\text { para solucionar luego sus conflictos, de esa } \\
\text { forma mejoran su convivencia sin afectar sus } \\
\text { rendimientos académicos. }\end{array}$ \\
\hline
\end{tabular}




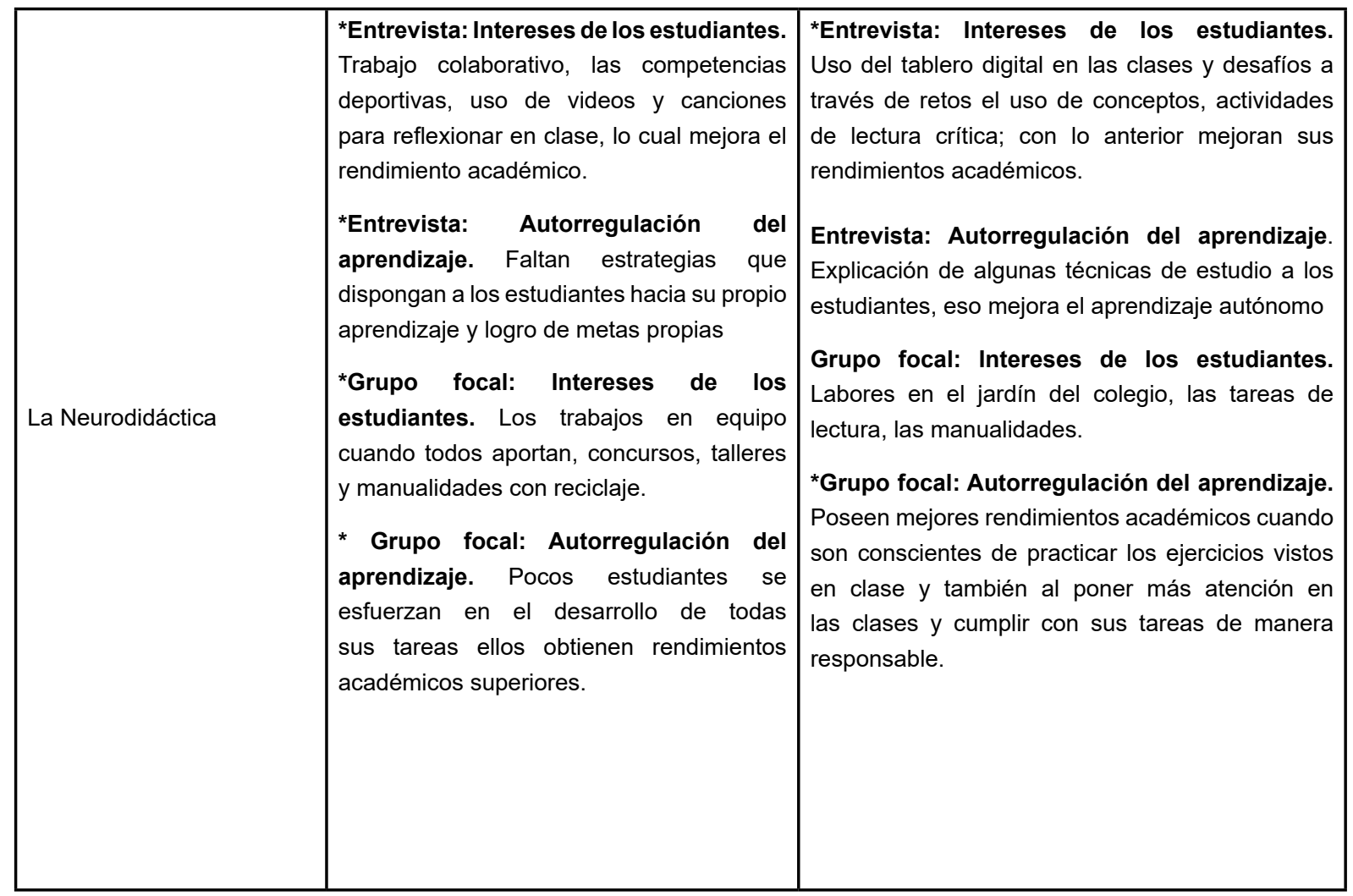

Fuente: Elaboración Propia

Cabe mencionar, que la información precedente, corresponde a factores internos, abordados en el trabajo de campo, desde los conceptos de la metacognición, inteligencia emocional y Neurodidáctica contemplados como estrategias de motivación académica, autoconcepto y aprendizaje autónomo.

Una vez analizados los hallazgos, se pudo establecer que los educadores pueden aportar al aprendizaje autorregulado y rendimiento académico desde el conocimiento de los avances y limitaciones que tienen los educandos para aprender, de ese modo, enseñarles pautas de auto seguimiento, autocontrol y autoevaluación, ofreciéndoles espacios de retroalimentación y reflexión crítica de los conocimientos adquiridos. Al respecto Flores y Gómez (2010) se refieren a la motivación como un indicador del rendimiento académico, como producto de un aprendizaje autónomo.
Visto de esa forma, para una adecuada autorregulación del aprendizaje en la sede A, habrá de orientarse desde la curiosidad del estudiantado, para que disfruten mientras aprenden. No obstante, en la sede $\mathrm{K}$ existen estrategias que los estudiantes reconocen como estimulantes del aprendizaje autónomo, permitiéndoles rendir académicamente. Así pues, se halla una correlación con lo afirmado por Garrido, Jiménez, Landa, y Páez (2013) quienes sostienen que la motivación académica conlleva al éxito escolar, de ahí que los buenos desempeños académicos son el producto de la motivación interna y externa de cada alumno.

\section{CONCLUSIONES}

- En el contexto sociocultural de la sede A y sede $\mathrm{K}$ de la Institución Educativa Cirales, existen factores externos que inciden en el rendimiento académico y aprendizaje autorregulado, tal es el caso del aspecto familiar caracterizado por la presencia de hogares disfuncionales, 
que conllevan al bajo autoconcepto y violencia intrafamiliar; situaciones reflejadas en la desmotivación académica y fracaso escolar.

-En las dos sedes muestreadas, los aspectos sociales que influyen en el rendimiento académico y aprendizaje autónomo son, la falta de transporte escolar y las dificultades para el desplazamiento en temporada de invierno. Sin embargo, los estudiantes de la sede A se encuentran en situación de vulnerabilidad frente a problemáticas relacionadas con el consumo de sustancias psicoactivas, donde a pesar de contar con apoyo de otras entidades, no ha sido posible establecer canales de comunicación lo suficientemente sólidos entre padres, estudiantes y docentes para conocer las causas $y$ formas como involucran personas ajenas a los escolares en dichas prácticas, aprovechando la falta de confianza en los hogares y problemas personales de los estudiantes.

- En lo referente a los aspectos externos de tipo escolar incidentes en el rendimiento académico, se pudo concluir que la desmotivación desde la parte personal y académica de algunos estudiantes; resultado de las condiciones del sector rural, limitan el acceso a la educación superior e influyen en el desánimo y falta de emprendimiento de los escolares, no encontrando el sentido propio de formarse y prepararse académicamente desde la primaria y la secundaria. Con base en lo anterior, se detectó que falta mayor compromiso y responsabilidad en la entrega de tareas escolares y el estudio para evaluaciones lo que conduce a la pérdida de materias, años académicos y deserción; dichos aspectos son más notorios en la sede $A$.

- De acuerdo con la comparación de los aspectos socioculturales de la sede $\mathrm{A}$ y sede $\mathrm{K}$ que inciden en el rendimiento académico, también se concluyó que falta de mayor control de los padres hacia los hijos en la sede $A$, convirtiéndose en un aspecto externo de tipo familiar que influye negativamente en el desempeño académico porque los estudiantes se distraen en sus noviazgos clandestinos y otras actividades de ocio como el celular sin ser supervisados por sus progenitores.

-A partir del análisis y comprensión de la información recolectada, se logró la descripción y comparación de los aspectos socioculturales de los estudiantes de la básica secundaria de la sede $\mathrm{A}$ y sede $\mathrm{K}$, así como la caracterización del rendimiento académico para dar paso a la formulación de estrategias que buscan la estimulación del aprendizaje autónomo, retroalimentación personalizada de los aprendizajes y la adecuación de espacios de reflexión crítica en el estudiantado.

Así pues, el mejoramiento del rendimiento académico se dará en la medida que la enseñanza que se imparta tenga en cuenta las limitaciones y ventajas que tiene particularmente cada estudiante en el momento de aprender y que ellos se centren en el autocontrol de sus aprendizajes asumiendo con responsabilidad su educación para la vida, desde la integralidad.

\section{RECOMENDACIONES}

La educación que se imparte hoy en día se ha venido reinventando como consecuencia de nuevos paradigmas y situaciones relacionadas con el autocuidado de cada miembro de las familias. Lo anterior, pone de manifiesto que, desde el trabajo colaborativo de docentes, padres de familia y estudiantes se deben establecer acciones hacia cambios curriculares pertinentes, especialmente en la educación básica y media, que ha mutado desde la presencialidad a la virtualidad, por un tiempo indefinido. Es provechoso como investigadores encontrar nuevos caminos hacia la formación en el aprendizaje autónomo ya que impacta el rendimiento académico y autodisciplina.

Es de suma importancia que las instituciones educativas tengan en cuenta el estudio de los 
aspectos socioculturales de sus estudiantes porque ellos marcan las realidades de las cuales emergen, el tipo de mediación docente y de los propios educandos, en la formación integral para la vida, en el marco de una educación humanizante que pueda afrontar los retos de la sociedad actual.

\section{REFERENCIAS BIBLIOGRÁFICAS}

Barreto, F., y Álvarez, J. (2017). Clima escolar y rendimiento académico en estudiantes de preparatoria. Daena: International Journal of Good Conscience, 12(2), 31-44.

Crespo, C., y Salamanca, A. (2007). El muestreo en la investigación cualitativa | NURE Investigación. Nure Investigación, 27, 1-4.

Flores, R., y Gómez, J. (2010). Un estudio sobre motivación hacia la escuela secundaria en estudiantes mexicanos. REDIE. Revista Electrónica de Investigación Educativa., 12(1), 22.

Garrido, M., Jiménez, N., Landa, A., y Páez, E. (2013). Factores que influyen en el rendimiento académico: La motivación como papel mediador en las estrategias de aprendizaje y clima escolar. ReiDoCrea, 2(2), 1-9.

Gutiérrez, J. (2014). El contexto familiar asociado al autoconcepto y al desempeño académico de los jóvenes de educación media de San Salvador. Revista entorno, (55), 41-50.

Hernández, R., Fernández, C., y Baptista, M. (2010). Metodología de la investigación. (Quinta edición.). México: Mc Graw Hill.

Hernández, V. (2017). Las competencias emocionales del docente y su desempeño profesional. Aalternativas en Psicología, 37, 7992.
Jama, V., y Cornejo, J. (2016). Influencia del aspecto socioeconómico en el rendimiento académico. Dominio de las Ciencias, 2(1), 102117.

Jurado, R. (2012). Condiciones socioculturales de la familia santandereana como escenario influyente en la deserción o permanencia escolar en preadolescentes de la Institución Educativa Campo Hermoso de Bucaramanga (Tesis de Maestría). Colombia: Universidad de la Sabana. Recuperado a partir de https://intellectum. unisabana.edu.co/handle/10818/8203

Luna, A. (2018). Factores internos y externos que influyen en el fracaso escolar de los estudiantes de tercero y cuarto del colegio rural Quiba alta. (Tesis de Maestría). Colombia: Universidad Externado de Colombia. Recuperado a partir de https://bdigital.uexternado.edu.co/ bitstream/001/1299/1/CBA-Spa-2018-Factores_ internos_y_externos_que_influyen_en_el_ fracaso_escolar_de_los_estudiantes_de_ tercero_y_cuarto_del_Colegio_Rural_Quiba_ Alta_Trabajo.pdf

Meneses, W., Morillo, S., Navia, G., y Grisales, M. (2013). Factores que afectan el rendimiento escolar en la institución educativa rural Las mercedes desde la perspectiva de los actores institucionales. Plumilla Educativa, 11(1), 433452. doi:10.30554/plumillaedu.11.364.2013

Muñoz, S., Ávila, W., y Grisales, M. (2014). Prácticas culturales y su influencia en el rendimiento académico. Plumilla Educativa, 13(1), 176-193. doi:10.30554/ plumillaedu.13.406.2014

Narváez, M., y Oyola, S. (2015). Influencia del contexto social en el rendimiento académico de los estudiantes del grado 7-1 de la institución educativa técnica Antonio Nariño Fe y Alegría de la Ciudad de Ibagué (Trabajo de grado). Colombia: Universidad del Tolima. Recuperado a partir de https://core.ac.uk/reader/81668648 
Parra, K. (2014). El docente y el uso de la mediación en los procesos de enseñanza y aprendizaje. Revista de investigación, 38(83), 155-180.

Quecedo, R., y Castaño, C. (2003). Introducción a la metodología de investigación cualitativa. Revista de Psicodidáctica, 14, 5-40.

Tilano, L., Henao, G., y Restrepo, J. (2009). Prácticas educativas familiares y desempeño académico en adolescentes escolarizados en el grado noveno de instituciones educativas oficiales del municipio de Envigado. El Agora USB, 9(1), 35-51.

Torrano, F., Fuentes, J., y Soria, M. (2017). Aprendizaje autorregulado: estado de la cuestión y retos psicopedagógicos. Perfiles Educativos, IISUE-UNAM, XXXIX(156), 160-173. 